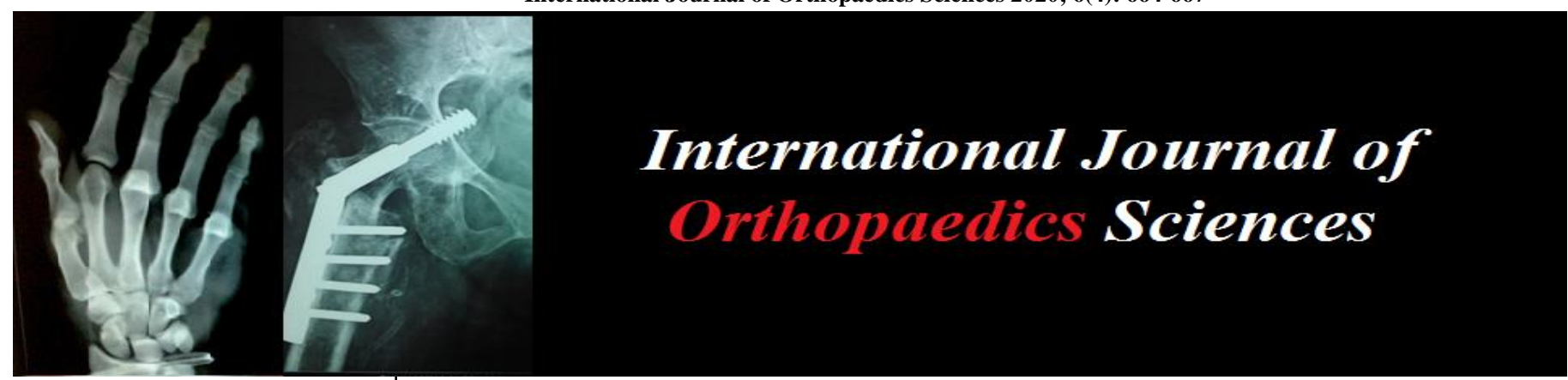

E-ISSN: 2395-1958

P-ISSN: 2706-6630

IJOS 2020; 6(4): 664-667

(C) 2020 IJOS

www.orthopaper.com

Received: 02-07-2020

Accepted: 09-08-2020

Dr. Abhishek Parashar

Bokaro General Hospital,

Bokaro Steel City, Jharkhand, India

Dr. Nikesh Panchbhai

Bokaro General Hospital,

Bokaro Steel City, Jharkhand,

India

Dr. Kumar Satyam

Bokaro General Hospital,

Bokaro Steel City, Jharkhand,

India

Dr. Masaji Nagargoje

Bokaro General Hospital,

Bokaro Steel City, Jharkhand,

India

Corresponding Author: Dr. Abhishek Parashar Bokaro General Hospital, Bokaro Steel City, Jharkhand, India

\section{Percutaneous pinning versus conservative management in distal radius fractures: A prospective analysis}

\author{
Dr. Abhishek Parashar, Dr. Nikesh Panchbhai, Dr. Kumar Satyam and \\ Dr. Masaji Nagargoje
}

DOI: https://doi.org/10.22271/ortho.2020.v6.i4j.2399

\section{Abstract}

Background: Fracture of the distal end of radius is one of the more common fractures encountered in the Orthopaedic practice. As longevity of the population increases and as accidents also increase, the incidence of this fracture is expected to increase. Achievement of good realignment of the fracture is essential from a functional and cosmetic point of view. The modalities for treatment of these fractures are varied and much confusion is present as regards appropriate treatment of various fracture types. A cost effective method of treatment is closed reduction and percutaneous pinning with K-wires. This study was conducted to analyze the outcome of Distal Radial Fractures treated with closed reduction and percutaneous pinning.

Methods: The present study was a prospective study of 60 cases of Distal Radius Fractures admitted to Bokaro General Hospital between the study period of November 2015 to October 2017. Cases were selected according to inclusion and exclusion criteria i.e., patients with distal radius fractures above the age of $18 \mathrm{yrs}$ and presenting within two weeks of injury. Medically unfit patients or those not willing for surgery or who presented with open fractures were excluded from the study.

Results: In our series of 60 cases there were 30 males and 30 females, with a maximum age of $72 y r s$, minimum age of $19 \mathrm{yrs}$ and an average age of 45 years. There was a no predominance of the side of the fracture. At the final follow up assessment with 'The Functional Assessment by The Gartland and Werley Criteria' 36 patients $(72 \%)$ achieved 'Excellent' result, 11 patients $(22 \%)$ achieved 'Good' result, 2 patients $(4 \%)$ achieved 'fair' result and 1 patient $(2 \%)$ achieved 'poor' result. Overall, $94 \%$ of the patients achieved an excellent or good result. On anatomical evaluation using 'Sarmiento's Criteria' 90\% of patients achieved a excellent or good result, while $10 \%$ achieved only a fair result.

Conclusion: Closed reduction and percutaneous pinning for distal radius fractures have shown to achieve good anatomical and functional outcome. The complications arising from the procedure were within acceptable limits. Further studies are required to assess its efficacy in various other fracture types.

Keywords: Distal radius fracture, percutaneous pinning, closed reduction, K- wires

\section{Introduction}

Fractures of the distal radius constitute one of the most common skeletal injuries treated by Orthopaedic surgeons. These injuries account for one sixth of all fractures evaluated in emergency room. Vast majority of fractures of distal radius are articular injuries that result in disruption of both radio-carpal and radio-ulnar joints. Better understanding of the spectrum of distal radius fractures has led to changing concepts of treatment. The optimal method of obtaining and maintaining an accurate restoration of distal radial anatomy remains a topic of considerable controversy. Wide arrays of techniques, including closed, percutaneous and open methods of reduction and stabilization have been increasingly advocated as successful treatment. Fracture of the distal radius was first described by Pouteau (1783) and Colles (1814). Prominent among the concept is that optimal management of distal radius fracture requires differentiation of the relatively low energy metaphyseal injuries from the more violent injuries that disturb the articular surface. The articular injuries are more frequently comminuted and unstable, and therefore less suitable for traditional method of closed reduction and cast application. Without supplemental skeletal fixation, redis placement of the fracture commonly to its pre-reduction position is inevitable, resulting in malunion, limited range of motion, weakness, pain, Sudecks Osteodystrophy and post-traumatic arthritis ${ }^{[1]}$. 
In our study we intend to analyze the functional and radiological outcome of distal radial fractures treated with closed reduction and percutaneous pinning.

\section{Materials and Methods}

This is a prospective study which includes cases of distal radial fractures treated with closed reduction and percutaneous pinning and closed reduction and casting at the Bokaro General Hospital, Bokaro Steel City Jharkhand between November 2015 and October 2017. The patients were followed up for an average period of 6 months. During the follow up X-rays were taken and the patients were assessed. We included patients above 18 years with fracture of the distal radius and those presented within 2 wks of injury. Patients with a Gustillo-Anderson grading greater than 1, fractures which require open reduction/ligamentotaxis (external fixator) were excluded from the study.

Post-operative pain and inflammation were managed using anti-inflammatory analgesics. Patients treated with percutaneous $\mathrm{k}$-wire were given intravenous cefuroxime $1 \mathrm{gm}$ twice daily for 2 days. After which pin site dressing was done and the patient discharged and was given oral antibiotics like cefixme $200 \mathrm{mg}$ twice daily for 5 days. Affected limb was kept elevated and patients were asked to perform active finger movements, elbow, shoulder movements from day one. Immediate post-operative check x-rays were taken in both PA and lateral views. The reduction of the fracture was confirmed and any displacements were noted. Patients were discharged after two days with routine pop instructions with active finger, elbow and shoulder exercises and followed up after 15 days. All the cases were followed up weekly intervals and assessed for displacement radio logically. After 6 weeks $\mathrm{k}$-Wires and cast removed and patient was assessed clinically for fracture union, range of movements and radio logically for parameters, physiotherapy was advised. After 6 weeks, regular follow-up was done at an interval of 3 months and 6 months respectively.

Methods of measuring $[2,3,4,5]$

A reference line is drawn along the central longitudinal axis of the radius on both AP and lateral x-rays.

\section{Measurement on the AP radiography \\ 1. Radial length}

To determine radial length 2 lines perpendicular to the centre line of the radius are drawn on AP view, one at the level of the distal end of the ulna not including the ulnar styloid process and the other at the level of the tip of radial styloid. The perpendicular distance between these two parallel lines measured in millimeter is the radial length. Normal $12 \mathrm{~mm}$ (range $10-13)$.

\section{Radial tilt or deviation or radial angulation}

A line perpendicular to the centre line of the radius is drawn on the AP view at the level of distal articular surface of ulna, another line drawn along the articular surface of radius. The angle formed by these 2 lines is the radial tilt. Normal $23^{\circ}$ range $\left(16^{\mathrm{O}}-30^{\circ}\right)$

\section{Radial shift or radial width}

This is measured on the AP view which is the distance between the longitudinal axis through the centre of the radius and the most lateral point of the radial styloid process.
4. Measurement on the lateral radiograph

One line is drawn perpendicular to the centre line of the radius on the lateral view in the region of the fracture and another line in drawn parallel to the distal radial articular surface. The angle formed by these 2 lines is the volar dorsal tilt. If the articular surface showed a volar tilt it's a positive angle. If there was a dorsal tilt then negative angle. Normal $11^{\circ}$ (range minus $2^{\circ}$ to plus 28 ). ${ }^{\circ}$

\section{Dorsal shift or displacement}

It is the distance between the central longitudinal axis of the radius to the most dorsal point of the distal end of the bone

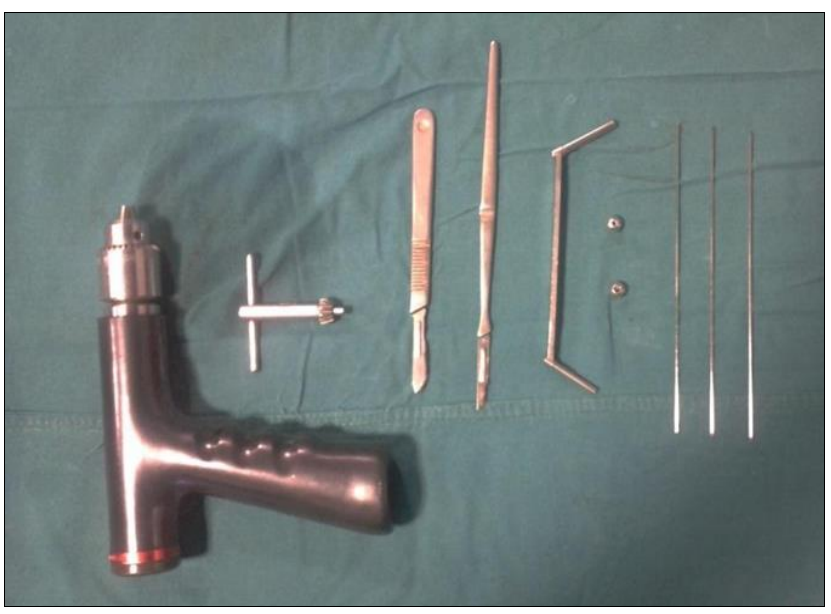

Fig 1: Instrumentation

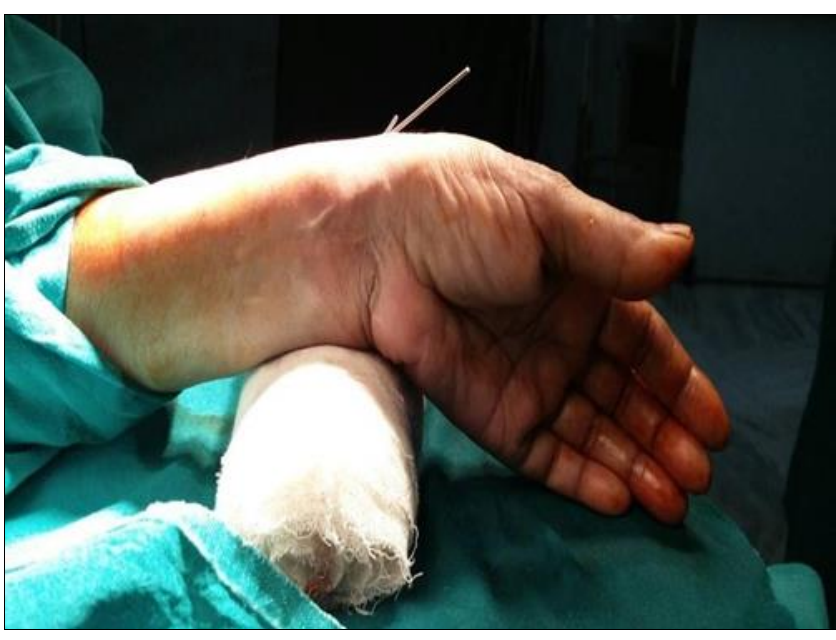

Fig 2: Patient's arm on the side table

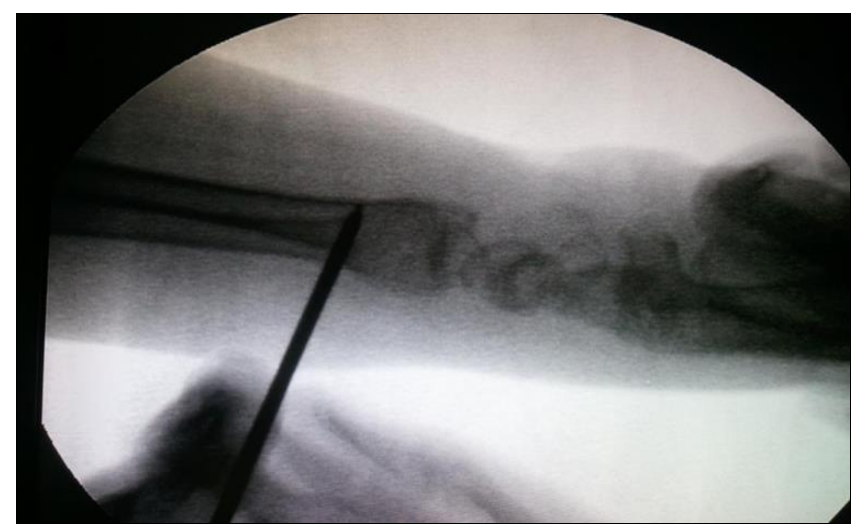

Fig 3: The kapandje technique for reduction 


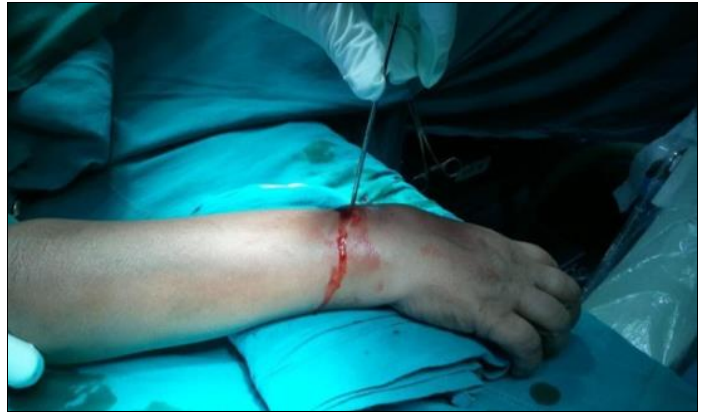

Fig 4: The kapandje technique for reduction

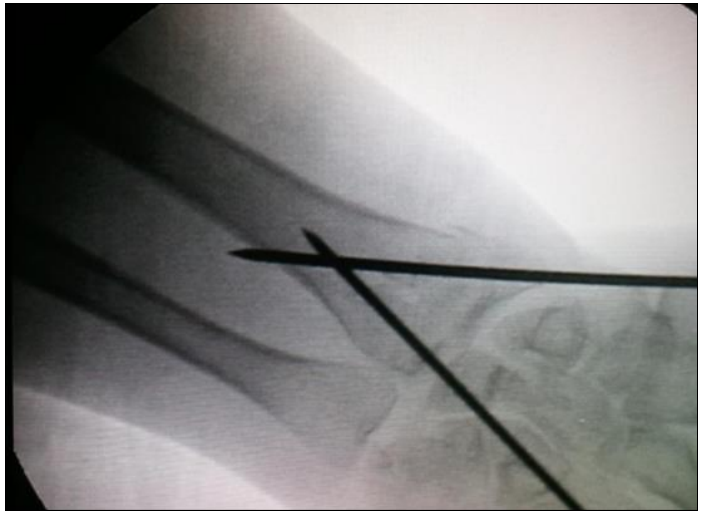

Fig 5: Insertion of k-wires under c-arm guidance

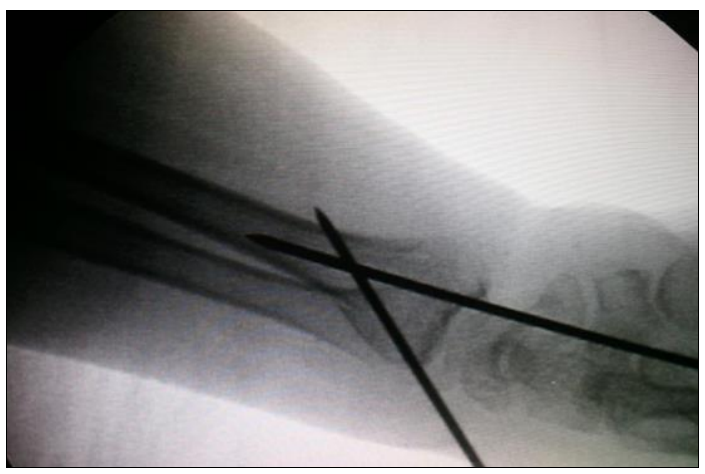

Fig 6: The lateral view for confirmation of position

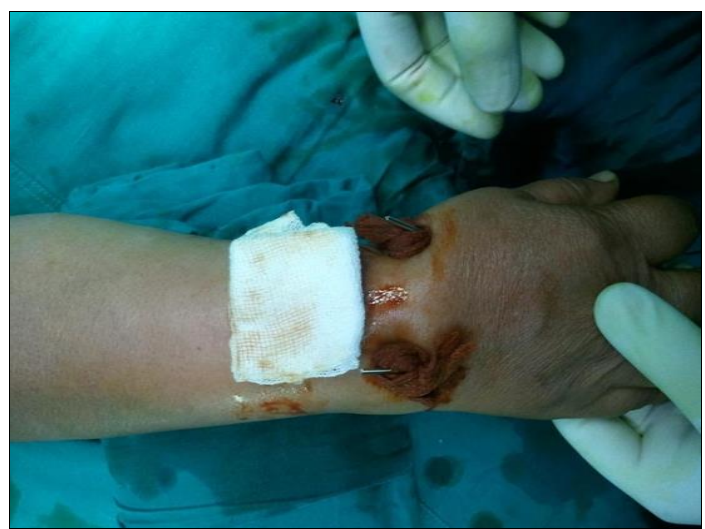

Fig 7: K-wires bent and dressing apllied

\section{Results}

Anatomical analysis of the data was done using Sarmiento's Modification of Lidstrom criteria. Functional Analysis of the Data was done using the Demerit point system of Gartland \& Werley with Sarmiento et al. Modification.

Table 1: End result point ranges

\begin{tabular}{|c|c|}
\hline Excellent & $0-2$ \\
\hline Good & $3-8$ \\
\hline Fair & $9-20$ \\
\hline Poor & 21 and above \\
\hline
\end{tabular}

There were 60 patients in the study, 30 in closed reduction with casting group and 30 in closed reduction with percutaneous $\mathrm{k}$ wire and casting group. The analysis of the mean age of patients was 54 years. Gender distribution in each group were equal i.e. total number 60 cases. The mechanism of injury in most cases was fall on out stretched hand $(85 \%)$. Majority of the patients $(85 \%)$, sustained the injury due to a fall. In group treated by closed reduction with casting, 16 patients $(27 \%)$ had right distal radius fracture and 14 patients $(23 \%)$ had left distal radius fracture. In group treated by closed reduction with $\mathrm{k}$ wire, 18 patients $(30 \%)$ had right distal radius fracture and 12 patients $(20 \%)$ had left distal radius fracture. Majority of the patients $(75 \%)$ who participated in our study did not have associated injuries.

According to Fernandez classification $60 \%$ of the patients in the study fell into type I fracture. About $35 \%$ came under the Type III fracture pattern. Based on AO classification $58 \%$ of patients had a Type A fracture pattern, $32 \%$ had a Type B and $10 \%$ had a Type C.

Complications are seen more in closed reduction with casting group when compared to closed reduction with K-Wire group.

Table 2: Show the closed reduction with K-wire and casting

\begin{tabular}{|c|c|c|}
\hline Movement & $\begin{array}{c}\text { Closed Reduction } \\
\text { with casting (mean } \\
\text { in degrees) }\end{array}$ & $\begin{array}{c}\text { Closed reduction with K- } \\
\text { Wire and casting (Mean in } \\
\text { degrees) }\end{array}$ \\
\hline Palmar Flexion & 63 & 65.5 \\
\hline Dorsiflexion & 61 & 62.65 \\
\hline Supination & 64 & 69.4 \\
\hline Pronation & 61 & 61.9 \\
\hline Ulnar Deviation & 22 & 24 \\
\hline Radial Deviation & 16 & 17.3 \\
\hline
\end{tabular}

There was statistically significant difference in range of movements between both groups. Patients treated with percutaneous K-Wire and casting group had significantly increased range of movements.

Radiological parameters were assessed for pre reduction, post reduction, 6 weeks and 3 months. There was significant difference in all the three parameters i.e, Volar tilt, Radial length, Radial inclination between both groups at the end of 3 months. Closed reduction with K-Wire group had shown not only better but also statistically significant anatomical reduction compared to other group.

Table 3: Radiological Measurement Closed Reduction with K- Wire

\begin{tabular}{|c|c|c|}
\hline Radiological Measurement & $\begin{array}{c}\text { Closed Reduction with casting } \\
\text { group (mean in degrees) }\end{array}$ & $\begin{array}{c}\text { Closed reduction with K- Wire and casting group } \\
\text { (Mean in degrees) }\end{array}$ \\
\hline Radial Length (mm) & 9.35 & 11.05 \\
\hline Volar Tilt (degrees) & 4.65 & 7.54 \\
\hline Radial Inclination (degrees) & 20.9 & 23.4 \\
\hline
\end{tabular}


Table 4: Subjective evaluation end of Result

\begin{tabular}{|c|c|c|}
\hline \multirow{2}{*}{ Result } & Subjective evaluation & End Result \\
\cline { 2 - 3 } & Number of Cases & Number of Cases \\
\hline Excellent & $38(63.0 \%)$ & $43(72.0 \%)$ \\
\hline Good & $17(28.0 \%)$ & $13(22.0 \%)$ \\
\hline Fair & $3(5.0 \%)$ & $3(4.0 \%)$ \\
\hline Poor & $2(4.0 \%)$ & $1(2.0 \%)$ \\
\hline
\end{tabular}

\section{Discussion}

Distal radius fracture is a common injury. The importance of anatomic reduction has been demonstrated by clinical studies as well as by laboratory assessment of force and stress studies. Accurate reduction of the fracture is the first step in treatment. Many options are available to maintain this initial reduction. The most common being closed reduction and cast application, but this is often associated with failure and complications ${ }^{[6]}$. External fixators cannot effectively maintain palmar tilt; also they also have been associated with complications as high as $60 \%{ }^{[7]}$. Percutaneous pinning with $\mathrm{K}$-wires was first recommended by Green ${ }^{[8]}$ as a simple and inexpensive procedure. The mean age of patients in our study was 44.98 (range 19-72) which is comparable to other studies published earlier ${ }^{[9]}$. As age advances there is osteoporosis and more chance of collapse of the fracture; also the elderly may have a harder time keeping to the rehabilitation protocol.

In this study as with other studies, there were marginally more men than women (30:20). This might be accounted for by the fact that more men were willing to undergo surgical intervention. The mode of injury was a fall in about 35 patients and a RTA in the other 15 patients. RTA's are generally associated with greater forces and therefore more severe fracture pattern. RTA as a cause for injury was more common in the younger age group in this study.

There was no statistical significance to the side involved in the fall. However one of the drawbacks that this study has is that the dominant hand was not included in the variables. This might have given a clearer picture as in other studies ${ }^{[9]} .10$ patients $(20 \%)$ of patients in our study had associated injuries. However patients with associated injuries in the same limb were not included in this study, as post op mobilization and thus scoring may have been compromised. Various studies have suggested that closed reduction and percutaneous pinning may not achieve a satisfactory result. Yet this study suggests that, satisfactory results may be achieved even with minimally comminuted fractures; provided adequate reduction can be achieved with closed reduction.

The functional results in this study are lower than the study by Abhishek K Das et al. ${ }^{[9]}$. However the reason for this could be that patients with extra-articular fractures with dorsal comminution and fractures with simple intra-articular fractures were also treated with percutaneous pinning. Also included in this study were a few patients with complex intraarticular fractures who were treated with pinning as closed reduction brought the major fragments into alignment and fracture reduction was maintained with the K-wires; also there were two patients who were unwilling for any intervention with plates or ex-fix. The results suggest that further research is needed to further evaluate this method of treatment in more complex fractures.

The anatomical outcome was evaluated using Sarmiento's modification of Lidstrom's criteria. The results of this study are comparable to the other studies that have been done previously. The comparison between the results of the functional outcome as evaluated by the Gartland \& Werley scoring system and the Anatomical outcome evaluated as mentioned above, confirm what other studies have previously shown, that the functional result need not mirror the anatomical evaluation. In this study there were nine cases of pin site infection. Four of which settled with antibiotics and the other five after removal of the K-wires. There was no persistence of the infection in any of the cases. Two of the cases with pin site infection experienced loosening of the pins. However the fracture progressed to healing satisfactorily. One of the patients with pin tract infection developed Reflex Sympathetic Dystrophy. As with other studies before, no significant cause for the same could be elicited. However patient did not mobilize her wrist or fingers post-surgery and she was immobilized for a period of 6 weeks as fracture did not show signs of adsfequate union.

\section{Conclusion}

Closed reduction and percutaneous pinning for reducible, unstable distal radius fractures have shown to achieve good anatomical and functional outcome. The complications arising from the procedure were within acceptable limits. The morbidities arising from prolonged anesthesia were also avoided. Also the financial impact to the patient was less as compared with other modes of operative treatment.

Further studies are required to assess its efficacy in various other fracture types.

\section{References}

1. David Litchman M. A Herbert Alexander. The Wrist and its Disorder. $2^{\text {nd }}$ Ed. Saunders 1997.

2. Terry Canale S, James Beaty H. Campbell's Operative Orthopaedics. $11^{\text {th }}$ ed. Philadelphia. Mosby Elsevier 2007.

3. Collins DC. Management and rehabilitation of distal radius fractures. Orthop Clin North Am 1993;24(2):36578.

4. Dowling JJ, Sawyer B. Jr. Comminuted Colles' fractures. Evaluation of a method of treatment. J Bone Joint Surg Am 1961;43-A:657-68.

5. Szabo RM. Extra-articular fractures of the distal radius. Orthop Clin North Am 1993;24(2):229-37.

6. Jupiter JB, Ring D, Weitzel PP. Surgical treatment of redisplaced fracture of the distal radius in patients older than 60 years. J Hand Surg Am 1994;19:325-40.

7. Gausephol T, Pennig D, Mader K. Principles of external fixation and supplementary technique in distal radius fractures. Injury 2000;31:56-70.

8. Green DP. Pins and plaster treatment of comminuted fractures of distal end radius. J Bone Joint Surg Am 1975;57:304-10.

9. Abhishek Das K, Nandkumar Sundaram, Thiruvengita G. Prasad. Percutaneous pinning for non-comminuted extraarticular fractures of distal radius. Indian $\mathrm{J}$ Orthop 2011;45(5):422-6. 